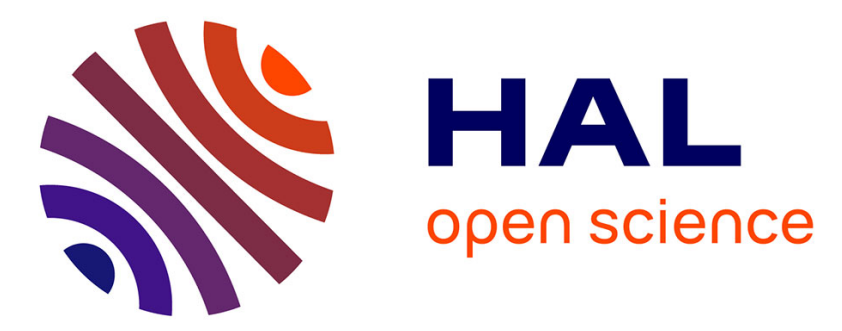

\title{
Supraclavicular recurrence after early breast cancer: a curable condition?
}

\author{
Anders N. Pedersen, Susanne Møller, Karina D. Steffensen, Vera Haahr, \\ Merete Jensen, Mette M. Kempel, Søren L. Jepsen, Ebbe L. Madsen, Anne \\ Roslind, Erik Sandberg, et al.
}

\section{To cite this version:}

Anders N. Pedersen, Susanne Møller, Karina D. Steffensen, Vera Haahr, Merete Jensen, et al.. Supraclavicular recurrence after early breast cancer: a curable condition?. Breast Cancer Research and Treatment, 2010, 125 (3), pp.815-822. 10.1007/s10549-010-0918-8 . hal-00561318

\section{HAL Id: hal-00561318 https://hal.science/hal-00561318}

Submitted on 1 Feb 2011

HAL is a multi-disciplinary open access archive for the deposit and dissemination of scientific research documents, whether they are published or not. The documents may come from teaching and research institutions in France or abroad, or from public or private research centers.
L'archive ouverte pluridisciplinaire HAL, est destinée au dépôt et à la diffusion de documents scientifiques de niveau recherche, publiés ou non, émanant des établissements d'enseignement et de recherche français ou étrangers, des laboratoires publics ou privés. 


\section{Supraclavicular recurrence after early breast cancer: \\ a curable condition?}

Anders N. Pedersen ${ }^{a^{*}}$, Susanne Møller ${ }^{b}$, Karina D. Steffensen ${ }^{c}$, Vera Haahr ${ }^{d}$, Merete Jensen ${ }^{e}$, Mette M. Kempel ${ }^{f}$, Søren L. Jepsen ${ }^{g}$, Ebbe L. Madsen ${ }^{h}$, Anne Roslind', Erik Sandberg ${ }^{j}$, Claudia Schöllkopf ${ }^{k}$, Peter G. Sørensen', Karen Margrethe Windfe ldt ${ }^{m}$, Michael Andersson ${ }^{a}$

aDepartment of Oncology, Rigshospitalet, Copenhagen, Denmark

${ }^{b}$ Danish Breast Cancer Cooperative Group, Rigshospitalet, Copenhagen, Denmark

${ }^{\mathrm{C} D e p a r t m e n t ~ o f ~ O n c o l o g y, ~ V e j l e ~ H o s p i t a l, ~ V e j l e, ~ D e n m a r k ~}$

${ }^{\text {d} D e p a r t m e n t ~ o f ~ O n c o l o g y, ~ V i b o r g ~ H o s p i t a l, ~ V i b o r g, ~ D e n m a r k ~}$

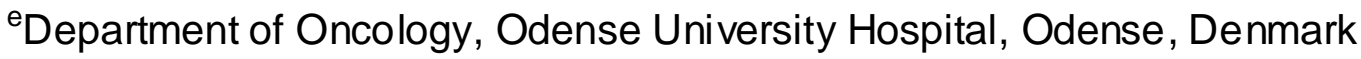
${ }^{f}$ Department of Oncology, Aalborg University Hospital, Aalborg, Denmark ${ }^{9}$ Department of Oncology, Aarhus University Hospital, Aarhus, Denmark hDepartment of Oncology, Sønderborg Hospital, Sønderborg, Denmark 'Department of Oncology, Herlev University Hospital, Herlev, Denmark 'Department of Oncology, Sydvestjyske Sygehus - Esbjerg , Esbjerg, Denmark kDepartment of Oncology, Hillerød Hospital, Hillerød, Denmark 'Department of Oncology, Roskilde Hospital, Roskilde, Denmark mDepartment of Oncology, Herning Hospital, Herning, Denmark *corresponding author; tel: +45 35450605; fax: +45 35456966; e-mail address: anders.navrsted.pedersen@rh.regionh.dk 


\section{Abstract}

Purpose:The prognosis of ipsilateral supraclavicular lymph node recurrence after early breast cancer appears to be worse than for other loco-regional recurrences, but better than for distant metastases. The purpose of the present study was to investigate the relationship between different types of salvage treatment and primary patient characteristics, treatment response, and survival after supraclavicular recurrence (SR) in a large patient population.

Methods: From the Danish Breast Cancer Cooperative Group treatment database 1977-2003, 305 patients were identified with SR without distant disease as site of first recurrence. Salvage treatment types as well as other factors were related to response and survival.

Results: The median follow-up time for progression after SR was 25 months. Complete remission was $76 \%$ among patients receiving excision surgery, $67 \%$ with combined loco-regional and systemic therapy, and $48 \%$ with systemic therapy alone. Median progression-free survival (PFS) and overall survival was 18 and 29 months, respectively. The 5 -year PFS probability was $15 \%$. In univariate analysis, combination salvage therapy, negative nodal status, and low malignancy grade were related to longer PFS. In multivariate analysis, salvage therapy and malignancy grade remained independent factors for survival. Conclusions: The prognosis of SR is generally poor. However, it appears to be a curable condition. An independent marker of improved outcome is local and systemic combination salvage treatment, which can be considered. 
Key words

breast cancer, prognosis, recurrence, supraclavicular, therapy 


\section{Introduction}

Loco-regional recurrence after primary early stage breast cancer occurs in up to $20 \%$ of patients. The most common locations are chest wall, breast and ipsilateral axillary lymph nodes. Recurrence in these sites is curable.

Supraclavicular recurrence (SR) is an unusual event with uncertain curability and consequently an undefined treatment approach. In general, this condition portends systemic metastases and poor survival.

Recently, a study [1] investigated the prognosis of different loco-regional recurrences among a group of patients originally enrolled in an adjuvant treatment trial by the Danish Breast Cancer Cooperative Group (DBCG): for the merged group of patients with ipsilateral infraclavicular and/or supraclavicular metastases, the authors found an intermediate prognosis, being poorer than for other loco-regional recurrence sites, but better than for distant metastases. Three studies of small patient cohorts with SR receiving multi-modality treatment have proposed a 5-year progression-free survival rate of up to $20 \%$ [2-4].

Little information exists about the influence of primary patient characteristics and of treatment modality on the prognosis of SR. The choice of treatment is controversial and primarily based on institutional preferences. Most information has been derived from retrospective studies. In case of limited local therapy the risk of progression is extremely high, and even with the addition of systemic treatment the rate of persistent tumour control remains low [5]. Few studies have investigated the outcome of radical local salvage treatment in 
combination with aggressive systemic therapy [2]. More data is needed to help clarify this complex situation, in order to decide the proper treatment approach. We have performed a retrospective study of 305 Danish patients with SR as presentation of first recurrence, relating primary patient characteristics and treatment of SR with treatment response, time to progression, and overall survival after SR. 


\section{Patients and methods}

\section{Study cohort}

45854 patients with early stage breast cancer enrolled in the DBCG adjuvant treatment database [6] between January 1977 and December 2003 were considered at risk of SR. All patients had histologically proven breast cancer free of supraclavicular or distant disease at primary diagnosis and no evidence of residual disease after primary treatment. Among this population, 305 patients subsequently reported off-study with a diagnosis of SR were studied. SR was defined as first recurrence more than one month after primary diagnosis, in the ipsilateral supraclavicular lymph node region, with or without other loco-regional metastasis, but with no evidence of distant metastases within one month of recurrence. Table 1 profiles patient characteristics at primary diagnosis in the study group.

\section{Treatment}

Treatment at primary diagnosis according to national guidelines has previously been described [6]. In brief, the surgical procedure comprised a tumourectomy or modified radical mastectomy, and partial axillary dissection. Patients with a tumourectomy were offered postoperative breast radiotherapy, and for high-risk patients the postoperative treatment predominantly consisted of locoregional (including supraclavicular) megavoltage irradiation. Systemic treatment to highrisk groups predominantly consisted of chemotherapy with cyclophosphamide, 5- 
fluorouacil, and methotrexate or epirubicin, and/or endocrine therapy with primarily tamoxifen (Table 1).

Treatment for subsequent SR was carried out according to institutional preference, and included biopsy or radical surgery, involved field or loco-regional radiotherapy, anthracycline or other type chemotherapy, and aromatase or nonaromatase inhibitor endocrine therapy.

\section{Follow-up and data collection}

After primary diagnosis, patients were routinely followed minimum by history and physical examination twice yearly for five years and yearly for an additional five years [6]. After diagnosis of SR, follow-up was conducted as per the treating oncologists' discretion, typically quarterly. Follow-up continued until death or last date of hospital contact. Data registration of death was 1 October 2007. Staging procedures at time of SR typically included physical examination, chest X-ray, bone scan, and laboratory tests.

The long-term follow-up data collection source for the investigated population was the patient individual medical record. Extractions from the medical records included patient, tumour and therapy characteristics at the time of primary diagnosis, and characteristics of SR, including date, size, pathological confirmation, concomitant loco-regional disease extension, treatment, response to treatment, loco-regional progression, distant progression and death or last patient contact. 


\section{Endpoints and statistical analysis}

Time to recurrence was defined as time from date of primary diagnosis to diagnosis of SR. Progression-free survival (PFS) was defined as time from SR to any localization of progression. The overall survival (OS) was calculated from SR to death of any cause. Complete remission was defined as disappearance of detectable tumour at SR. Potential prognostic factors for survival after SR were analysed as to patient individual variables before, at, and after SR. Interactions between confounding factors were analysed with the Mantel-Haenszel test. The probability of survival was estimated by the Kaplan-Meier method, and frequencies were reported. The log-rank test was used for comparison between subgroups of patients. Time proportionality of hazard ratios $(\mathrm{HR})$ was evaluated by Schonfeldt analysis. All analysed univariate variables were included in a Cox regression model, and successively a stepwise backward elimination was performed. Level of significance was chosen as $5 \%$. 


\section{Results}

\section{Survey of the SR cohort}

Data was collected from a total of 305 breast cancer patients diagnosed with SR as first site of failure between 1977 and 2003. The frequency reported among patients entered into the DBCG adjuvant treatment database was as low as $1 \%$ (305/45854). A proportionally large fraction of the study group had axillary lymph node involvement $(74 \%)$ and - for ductal carcinomas - high tumour malignancy grade $(80 \%)$ at primary diagnosis (Table 1$)$. The median time to recurrence was 27 months (range 2-114 months). At time of SR diagnosis, the median and mean age was 57 years (range 32-79 years). Among the cohort, 188 patients had isolated SR and 117 patients had synchronous other loco-regional disease extension, mostly with only one additional disease site (86 patients). After SR, 234 (77\%) events of progression had been observed and 267 (88\%) patients were dead at the time of analysis. The median follow-up time after SR was 25 months (range 1-136 months) for progression and 43 months for death (range 2275 months).

$158(52 \%)$ patients had reported local symptoms prior to verification of SR. Median supraclavicular tumour size was $14 \mathrm{~mm}$ (range $4-70 \mathrm{~mm}$ ). $96 \%$ of the patients had biopsy verification of their recurrence; in the remaining select cases, the recurrence was verified by ultrasound, X-ray and/or physical examination. Additional staging procedures comprised physical examination, chest X-ray/CT, skeletal X-ray/CT or bone scan, and laboratory tests for $\geq 95 \%$ of 
the patients; liver ultrasound or CT was performed in $35 \%$ of the cases. Table 2 profiles patient characteristics at SR.

\section{Treatment of SR}

Most of the patients with SR received systemic salvage treatment, whereas a smaller fraction had loco-regional treatment, either as radiotherapy alone or in combination with surgery (Table 3 ).

$19 \%$ of the patients were surgically treated with at least removal of the macroscopic tumour. 103 (33\%) patients received radiotherapy with curative intent at least to the involved field with megavoltage to $48-50$ Gy in $22-25$ fractions. A boost of 10-20 Gy was applied in 56 of these cases. Only $10 \%$ of the patients had combined surgery and radiotherapy.

Endocrine treatment was used for $123(40 \%)$ of the patients, predominantly as tamoxifen (107 cases) and rarely as letrozol or megestrole acetate (16 cases). Virtually all of these patients (120) had a steroid hormone receptor positive tumour or unknown receptor status. Endocrine treatment was combined with loco-regional therapy in 52 cases. Chemotherapy was used for $136(45 \%)$ of the patients. Anthracycline based regimens were administered in 99 of these cases, whereas taxanes were used in only 4 cases. Chemotherapy was combined with loco-regional therapy in only 37 cases.

Overall, 78 (26\%) patients had combined loco-regional (surgery and/or radiation) and systemic (endocrine and/or chemo) therapy.

Since type of treatment at SR was not randomly assigned, but carried out according to institutional preference, the study group was further analysed for 
interactions between confounding factors and salvage treatment: patients with negative lymph node status at primary diagnosis received combination therapy at SR more often than patients with node positive primary diagnosis (42\% versus 20\%) $(\mathrm{P}=0.01)$. Explanatory, patients without adjuvant primary radiotherapy prevalently node negative cases - received local or combination salvage therapy more often than patients with adjuvant primary radiotherapy $(P=0.03)$. Also, an interaction between tumour size at SR and systemic or combination therapy was observed $(\mathrm{P}=0.0005)$. No interactions were observed for tumour size or grade at primary diagnosis, age or disease extension at recurrence, or recurrence-free interval.

\section{Response to treatment}

Overall, complete remission rate was $48 \%$, partial remission $23 \%$, stable disease $9 \%$, and early progressive disease during salvage treatment $20 \%$. Complete remission rates depended on type of treatment, and the highest frequency was seen in patients given loco-regional therapy; among patients receiving excision surgery complete remission rate was $76 \%$ - significantly higher than for patients not given surgery $(43 \%)(\mathrm{P}<0.0001)$ - and for curative radiotherapy the rate was $62 \%$. A small group of 15 patients receiving only surgery plus radiotherapy experienced a very high remission rate of $87 \%$. For patients receiving systemic therapy, the complete remission rate was lower, $58 \%$ for chemotherapy and only $42 \%$ for endocrine treatment. Combining loco-regional and systemic therapy resulted in a proportionally higher rate of remission by $67 \%$, as compared to loco- 
regional therapy only (64\%) or - in particular - systemic therapy only (40\%) $(\mathrm{P}<0.0001)$.

Analysis for interactions between confounding factors and salvage treatment response revealed a positive correlation between decreasing tumour size at $S R$ and rate of complete remission $(P=0.03)$. However, there was no interaction with response for primary tumour size, grade, lymph node status, age, recurrence-free interval, or whether SR had synchronous other loco-regional disease extensions or not.

\section{Prognosis after SR}

After treatment of SR, follow-up was conducted between 2 and 6 times yearly for $93 \%$ of the patients. Thus, treatment induced persistent tumour control could be assessed. 66 (22\%) patients remained free of evidence of disease during the entire follow-up period, whereas 138 ultimately progressed locally and 96 progressed with distant metastases. At time of analysis 31 (10\%) patients still remained alive and free of progression. Figure 1 shows the Kaplan-Meier estimates of PFS and OS after SR. The median PFS and OS was 18 and 29 months, respectively. The 5-year PFS and OS probability was 15 and 24\%, respectively (Table 4). Survival in relation to prognostic parameters is also shown in Table 4.

In univariate analysis, the following parameters were related to prognosis of SR: response to salvage treatment, where progression after SR was related to salvage treatment response - i.e. complete remission was related to good prognosis, whereas partial or no response predicted poor survival $(P<0.0001)$; 
type of salvage treatment, where systemic treatment resulted in prolonged progression-free survival $(\mathrm{P}=0.003)$ - in particular the risk of disease progression was significantly reduced when combined with loco-regional treatment as demonstrated in Figure $2(P=0.01)$; negative nodal status $(P=0.02)$ and low tumour grade $(P=0.03)$ at primary diagnosis were related to good SR prognosis. There was a longer OS for patients experiencing $S R$ after $>2$ years $(P=0.004)$. Multivariate Cox analysis included 280 patients with 233 events regarding PFS and 305 patients with 267 events regarding OS. The analysis demonstrated that salvage treatment assignment was an independent parameter for PFS (Table 5); for loco-regional versus combination treatment the hazard ratio was 1.8 (95\% confidence interval: 1.2-2.8) $(P=0.006)$. Also, negative lymph node status and low tumour malignancy grade at primary diagnosis remained independent parameters $(P=0.03)$. For $O S$, salvage treatment $(P=0.04)$ and malignancy grade $(P=0.02)$ were the only independent parameters.

\section{Subgroup analysis of 225 patients with positive lymph node status}

A separate exploratory analysis of the unique group of 225 patients with the high risk factor of positive lymph node status at primary diagnosis was performed, with the understanding that salvage treatment assignment and PFS was significantly different from the group with negative nodal status. After SR, 171 events of progression and 196 deaths had been observed. In univariate analysis, combination salvage treatment remained significantly associated with prolonged time to progression, indicating its robustness as a prognostic marker for PFS. Multivariate analysis confirmed salvage treatment assignment as an independent 
parameter for PFS with a hazard ratio of 1.5 (95\% confidence interval: $1.03-2)$

$(P=0.04)$ in favour of combination therapy. 


\section{Discussion}

In this study we have presented data on salvage treatment, response and prognosis of SR for the, to our knowledge, largest cohort so far published. However, due to the non-randomised retrospective nature of the study, type of treatment at SR was not randomly assigned, but individually selected according to institutional preference. Inevitable bias in the selection of treatment therefore limits the interpretation of the therapeutic options.

Basically, the risk of SR is low [7-9], as also indicated by the frequency reported among patients entered into the DBCG adjuvant treatment database of only $1 \%$ among unselected consecutive patients treated for early breast cancer. The completeness of the registration of SR as site of first recurrence in the DBCG database is unknown and some misclassification may have found place. Thus, the reported number is a minimum estimate. The risk of SR may be dependent on patient characteristics and primary treatment. Although our study did not aim at identifying risk factors for supraclavicular failure, it appears from the patient characteristics at primary diagnosis that a n expectedly large fraction of the study group presented with the high-risk parameters of axillary lymph node positive disease and high tumour malignancy grade as compared to the general patient population [6], in agreement with previous findings [9]. The median time to recurrence of 27 months did not differ significantly from other reports on locoregional recurrence $[1,2]$. The diagnostic set-up at time of SR of the presented cohort verified the recurrence as truly not disseminated. However, only a minority of the patients had CT, and newer modalities such as MR and PET were not 
included. It should be appreciated that consistent use of such modalities might have induced stage migration. Thus, in one study as many as $50 \%$ of patients with loco-regional failure had additional disease after thoracic CT [10].

Recurrences in the residual breast, surgical scar or ipsilateral axilla are usually aggressively approached with both local and systemic salvage the rapy, whereas SR traditionally has been offered systemic therapy only. This policy has been under change recently, based on small studies demonstrating long-term survival after multi-modality salvage therapy of SR [2-4]. In our study, the far majority of the patients received systemic salvage treatment $(75 \%)$, whereas a smaller fraction had curative loco-regional treatment, either as radiotherapy alone or in combination with surgery. The use of radiotherapy was restricted to patients not previously irradiated supraclavicularly. In this study loco-regional treatment resulted in a high complete remission rate. Severe morbidity associated with uncontrolled supraclavicular recurrence was thereby perhaps prevented. The addition of endocrine treatment after radical surgery and/or radiotherapy of a loco-regional recurrence prolongs PFS [11], and should be considered for patients with hormone sensitive tumours. Little information exists for the benefit of chemotherapy for loco-regional recurrence [12]. The present results are biased with respect to choice of salvage treatment since the choice could be influenced by other conditions such as age and comorbidity. Also, patients were not treated according to modern standards, i.e aromatase inhibitors, taxanes and newer targeted therapies were rarely used as salvage treatment. Furthermore, our study showed that patients with negative lymph node status at primary diagnosis received combination therapy at $\mathrm{SR}$ more often than patients with node positive 
primary diagnosis ( $42 \%$ versus $20 \%$ ), indicative of node positive patients already having received supraclavicular radiotherapy prior to SR. Also, an interaction between tumour size at SR and salvage therapy was observed: patients with small size tumours were more often offered local therapy only, indicative of suboptimal treatment resulting in high but not lasting rates of remission.

Despite the treatment inadequacies of $\mathrm{SR}$, complete remission was achieved in half of the cases, and early progression only occurred for 1 of 5 patients. The present study indicates that excision surgery or loco-regional megavoltage radiotherapy could improve local control; especially when combined radical radiotherapy and surgery was employed a high control rate was achieved.

Ultimately, in the present study the median survival after SR was short (29 months), but a remarkable proportion of the patients (22\%) remained free of evidence of disease during the entire follow-up period. Furthermore, a larger fraction of the patients escaped distant progression (45\%). Several factors, primary tumour as well as recurrence related, were found to be prognostic for survival. In the multivariate analysis, first of all systemic combination salvage treatment was an independent prognostic marker of prolonged PFS and OS, indicative of a higher efficiency than monotherapy. Also, nodal status and tumour grade at primary diagnosis were related to SR prognosis, indicating inherent aggressive tumour factors less sensitive to treatment. Conversely, there was a longer survival for patients experiencing late SR from slowly growing tumours which may respond longer to salvage treatment. Interpretation of this data must be performed with the understanding of the inherent bias in selection of patients for different strategies of salvage treatment. 
The prognosis of SR is generally poor: It has been shown that patients with SR have a 5-year survival ranging from anywhere between 5 and 35\% [1315], far different from the cure rate of $35-50 \%$ for an axillary recurrence [16-18]. Nielsen et al. [1] investigated the prognosis of different loco-regional sites as first recurrence among patients originally enrolled in an adjuvant treatment trial by the DBCG: for the merged group of patients with infraclavicular and/or supraclavicular metastases, the authors proposed an intermediate prognosis, being poorer than for other loco-regional recurrence sites, but better than for distant metastases. The three small studies using aggressive multi-modality treatment have demonstrated a 5-year disease-free survival rate of approximately $20 \%$ [2-4]. A recently published prospective study including supraclavicular recurrence $+/$ - other loco-regional manifestations demonstrated a similar survival rate [19].

Fewer patients with the high risk factor of positive lymph node status at primary diagnosis received local or combination salvage therapy than node negative patients - reflecting that salvage radiotherapy in this group was rarely an option due to prior primary adjuvant irradiation. Nevertheless, the prognostic power of salvage treatment type for survival after SR was also confirmed in this unique group of 225 node positive patients: combination treatment remained significantly associated with prolonged time to progression, indicating its robustness as a prognostic marker for PFS.

In this retrospective cohort study of SR without distant disease, combined loco-regional and systemic therapy was related to longer PFS and OS compared to systemic or loco-regional therapy alone. Radical surgery and/or high-dose 
radiotherapy seemed to be a mandatory part of the treatment in order to enhance persistent tumour control and systemic therapy in order to prevent distant metastases. However, the non-randomised nature of the study limits the interpretation of the therapeutic options; in the absence of randomised evidence and in spite of not up-to-date systemic treatments used in the study, a combined treatment approach could be considered in supraclavicular recurrent breast cancer. Thus, this rare site of recurrence is not inevitably a nidus for dissemination, and appears to be an occasionally curable condition. 


\section{References}

1. Nielsen H M, Overgaard M, Grau C et al (2006) Loco-regional recurrence after mastectomy in high-risk breast cancer--risk and prognosis. An analysis of patients from the DBCG 82 b\&c randomization trials. Radiother Oncol 79:147-155

2. Chen S C, Chang H K, Lin Y C et al (2006) Prognosis of breast cancer after supraclavicular lymph node metastasis: not a distant metastasis. Ann Surg Oncol 13:1457-1465

3. Pergolizzi S, Settineri N, Santacaterina A et al (2001) Ipsilateral supraclavicular lymph nodes metastases from breast cancer as only site of disseminated disease. Chemotherapy alone vs. induction chemotherapy to radical radiation therapy. Ann Oncol 12:1091-1095

4. van der Sangen M J, Coebergh J W, Roumen R M et al (2003) Detection, treatment, and outcome of isolated supraclavicular recurrence in 42 patients with invasive breast carcinoma. Cancer 98:11-17

5. Ampil F L, Caldito G, Li B D and Burton G V (2003) Supraclavicular nodal relapse of breast cancer: prevalence, palliation, and prognosis. Eur $\mathrm{J}$ Gynaecol Oncol 24:233-235

6. Moller S, Jensen M B, Ejlertsen B et al (2008) The clinical database and the treatment guidelines of the Danish Breast Cancer Cooperative Group 
(DBCG); its 30-years experience and future promise. Acta Oncol 47:506524

7. Chen S C, Chen M F, Hwang T L et al (2002) Prediction of supraclavicular lymph node metastasis in breast carcinoma. Int J Radiat Oncol Biol Phys $52: 614-619$

8. Recht A, Gray R, Davidson N E et al (1999) Locoregional failure 10 years after mastectomy and adjuvant chemotherapy with or without tamoxifen without irradiation: experience of the Eastern Cooperative Oncology Group. J Clin Oncol 17:1689-1700

9. Livi L, Scotti V, Saieva C et al (2010) Outcome After Conservative Surgery and Breast Irradiation in 5,717 Patients with Breast Cancer: Implications for Supraclavicular Nodal Irradiation. Int J Radiat Oncol Biol Phys 76:978983

10. Cheng J C, Cheng S H, Lin K J et al (1998) Diagnostic thoracic-computed tomography in radiotherapy for loco-regional recurrent breast carcinoma. Int J Radiat Oncol Biol Phys 41:607-613

11. Waeber M, Castiglione-Gertsch M, Dietrich D et al (2003) Adjuvant therapy after excision and radiation of isolated postmastectomy locoregional breast cancer recurrence: definitive results of a phase III randomized trial (SAKK 23/82) comparing tamoxifen with observation. Ann Oncol 14:1215-1221 
12. Haylock B J, Coppin C M, Jackson J et al (2000) Locoregional first recurrence after mastectomy: prospective cohort studies with and without immediate chemotherapy. Int J Radiat Oncol Biol Phys 46:355-362

13. Fodor J, Toth J, Major T et al (1999) Incidence and time of occurrence of regional recurrence in stage I-II breast cancer: value of adjuvant irradiation. Int J Radiat Oncol Biol Phys 44:281-287

14. Halverson K J, Perez C A, Kuske R R et al (1992) Survival following locoregional recurrence of breast cancer: univariate and multivariate analysis. Int J Radiat Oncol Biol Phys 23:285-291

15. Kiricuta I C, Willner J, Kolbl O and Bohndorf W (1994) The prognostic significance of the supraclavicular lymph node metastases in breast cancer patients. Int J Radiat Oncol Biol Phys 28:387-393

16. Abraham R, Nagy T, Goss P E and Crump M (2000) High dose chemotherapy and autologous blood stem cell support in women with breast carcinoma and isolated supraclavicular lymph node metastases. Cancer 88:790-795

17. Clemons M, Hamilton T, Mansi J et al (2003) Management of recurrent locoregional breast cancer: oncologist survey. Breast 12:328-337

18. Singletary S E, Allred C, Ashley P et al (2002) Revision of the American Joint Committee on Cancer staging system for breast cancer. J Clin Oncol 20:3628-3636 
19. Pergolizzi S, Adamo V, Russi E et al (2006) Prospective multicenter study of combined treatment with chemotherapy and radiotherapy in breast cancer women with the rare clinical scenario of ipsilateral supraclavicular node recurrence without distant metastases. Int J Radiat Oncol Biol Phys $65: 25-32$ 


\section{Tables}

Table 1 Patient characteristics and treatment at primary diagnosis

\begin{tabular}{ll}
\hline Parameter & Frequency \\
\end{tabular}

All patients

Tumour size

$\leq 20 \mathrm{~mm}$

$>20 \mathrm{~mm}$

Unknown

Positive lymph nodes

0

$1-3$

$>3$

Unknown

Histology

Ductal carcinoma

Non-ductal carcinoma

Malignancy grade (ductal only)

I

II

III
$305(100 \%)$

$118(39 \%)$

$177(58 \%)$

$10(3 \%)$

$79(26 \%)$

$84(28 \%)$

$141(46 \%)$

1

$276(90 \%)$

$29(10 \%)$

$44(16 \%)$

$145(53 \%)$

$76(27 \%)$ 
Unknown

Hormone receptor status

Negative

Positive

Unknown

Chemotherapy

No/unknown

Yes

Hormonal therapy

No/unknown

Yes

Radiotherapy

No/unknown

Yes
$11(4 \%)$

$94(31 \%)$

$140(46 \%)$

$71(23 \%)$

$151(50 \%)$

$154(50 \%)$

$189(62 \%)$

$116(38 \%)$

235 (77\%)

$70(23 \%)$ 
Table 2 Patient characteristics at supraclavicular recurrence

\begin{tabular}{|c|c|}
\hline Parameter & Frequency \\
\hline All patients & $305(100 \%)$ \\
\hline \multicolumn{2}{|l|}{ Age } \\
\hline$\leq 50$ years & $85(28 \%)$ \\
\hline $51-60$ years & $91(30 \%)$ \\
\hline$>60$ years & $129(42 \%)$ \\
\hline \multicolumn{2}{|c|}{ Supraclavicular tumour size } \\
\hline$\leq 10 \mathrm{~mm}$ & $90(30 \%)$ \\
\hline $11-20 \mathrm{~mm}$ & 103 (34\%) \\
\hline$>20 \mathrm{~mm}$ & $59(19 \%)$ \\
\hline Unknown & $53(17 \%)$ \\
\hline \multicolumn{2}{|c|}{ Synchronous sites } \\
\hline No & $188(62 \%)$ \\
\hline Yes & $117(38 \%)$ \\
\hline \multicolumn{2}{|l|}{ Symptoms } \\
\hline No & $146(48 \%)$ \\
\hline Yes & $158(52 \%)$ \\
\hline Unknown & 1 \\
\hline \multicolumn{2}{|l|}{ Verification } \\
\hline Histology & $82(28 \%)$ \\
\hline Cytology & $190(65 \%)$ \\
\hline
\end{tabular}


Other

Time to recurrence

$\leq 2$ years

$>2$ years
$12(4 \%)$

132 (43\%)

173 (57\%) 
Table 3 Treatment of supraclavicular recurrence

\begin{tabular}{ll}
\hline Modality & Frequency \\
\hline Excision surgery & $19 \%$ \\
Curative radiotherapy & $33 \%$ \\
Surgery+radiotherapy & $10 \%$ \\
Endocrine therapy & $40 \%$ \\
Chemotherapy & $45 \%$ \\
Endocrine+chemotherapy & $11 \%$ \\
No systemic therapy & $25 \%$ \\
Systemic therapy only & $49 \%$ \\
Local+systemic therapy & $26 \%$ \\
\hline
\end{tabular}


Table 4 Five-year progression-free and overall survival probabilities after supraclavicular recurrence

\section{Progression-free survival}

Overall survival

Parameter Probability P-value Probability P-value

\begin{tabular}{|c|c|c|c|c|}
\hline All patients & 15 & & 24 & \\
\hline Age & & 0.89 & & 0.97 \\
\hline$\leq 50$ years & 15 & & 24 & \\
\hline $51-60$ years & 15 & & 23 & \\
\hline$>60$ years & 15 & & 24 & \\
\hline Primary tumour size & & 0.31 & & 0.91 \\
\hline$\leq 20 \mathrm{~mm}$ & 15 & & 23 & \\
\hline$>20 \mathrm{~mm}$ & 13 & & 25 & \\
\hline Malignancy grade & & 0.03 & & 0.004 \\
\hline 1 & 13 & & 29 & \\
\hline$\|$ & 15 & & 30 & \\
\hline III & 7 & & 12 & \\
\hline Positive lymph nodes & & 0.02 & & 0.03 \\
\hline 0 & 17 & & 29 & \\
\hline $1-3$ & 9 & & 22 & \\
\hline$>3$ & 11 & & 23 & \\
\hline Time to recurrence & & 0.21 & & 0.004 \\
\hline$\leq 2$ years & 15 & & 19 & \\
\hline
\end{tabular}


$>2$ years

Supraclavicular tumour size

$\leq 10 \mathrm{~mm}$

$11-20 \mathrm{~mm}$

$>20 \mathrm{~mm}$

Synchronous sites

No

Yes

Local salvage treatment

No

Yes

Systemic salvage treatment

No

Yes

Combination salvage treatment

Local + systemic

20

11

7

0.64

8

14

14

0.67

15

13

0.9

10

10

0.003

7

12

0.01

Systemic only

Local only
37

0.91

21

24

28

0.88

26

23

0.35

20

29

18

26

33

21

18 
Table 5 Independent parameters for survival after supraclavicular recurrence

\begin{tabular}{|c|c|c|c|c|}
\hline \multirow[b]{2}{*}{ Parameter } & \multicolumn{2}{|c|}{ Progression-free survival } & \multicolumn{2}{|c|}{ Overall survival } \\
\hline & $P$-value & $\mathrm{HR}(95 \% \mathrm{Cl})$ & P-value & $\mathrm{HR}(95 \% \mathrm{Cl})$ \\
\hline Salvage treatment & 0.006 & & 0.04 & \\
\hline Local + systemic & & 1.0 & & 1.0 \\
\hline Systemic only & & $1.3(0.9-1.8)$ & & $1.2(0.9-1.7)$ \\
\hline Local only & & $1.8(1.2-2.8)$ & & $1.5(1.1-2.3)$ \\
\hline Malignancy grade & 0.03 & & 0.02 & \\
\hline 1 & & 1.0 & & 1.0 \\
\hline$\|$ & & $1.0(0.8-1.4)$ & & $1.1(0.8-1.5)$ \\
\hline III & & $1.6(1.1-2.3)$ & & $1.6(1.1-2.2)$ \\
\hline Lymph node status & 0.03 & & & \\
\hline 0 & & 1.0 & & \\
\hline 1 & & $1.5(1.0-2.2)$ & & \\
\hline$>3$ & & $1.5(1.1-2.2)$ & & \\
\hline
\end{tabular}

HR: Hazard Ratio; CI: Confidence Interval 


\section{Figure Captions}

Fig. 1 Progression-free (a) and overall survival (b) after supraclavicular recurrence

Fig. 2 Progression-free survival by combination of salvage treatment after supraclavicular recurrence. Note that in this non-randomised retrospective cohort study type of treatment was not randomly assigned, but individually selected according to institutional preference! Syst+loc: combined systemic and local therapy; Syst: systemic therapy alone; No syst: no systemic therapy

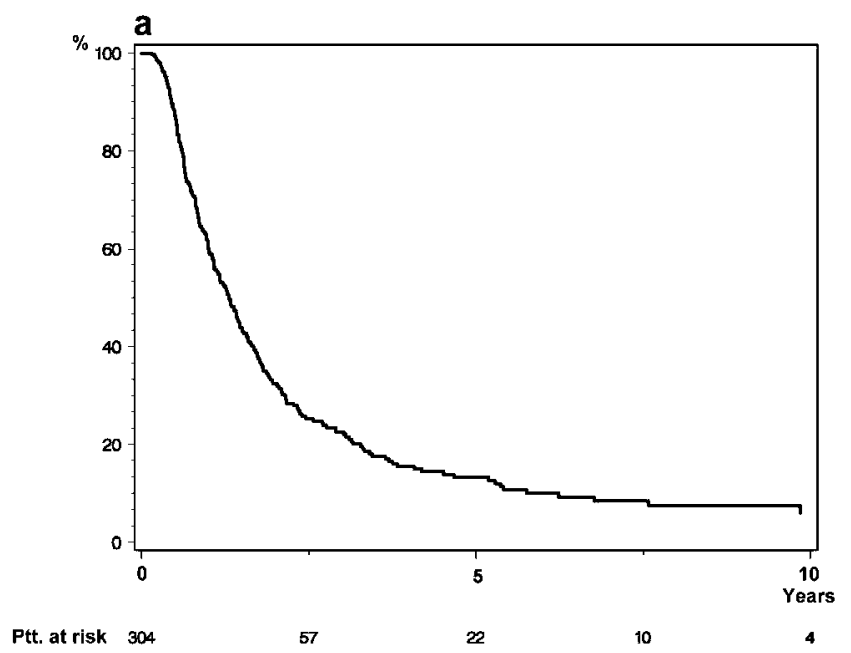



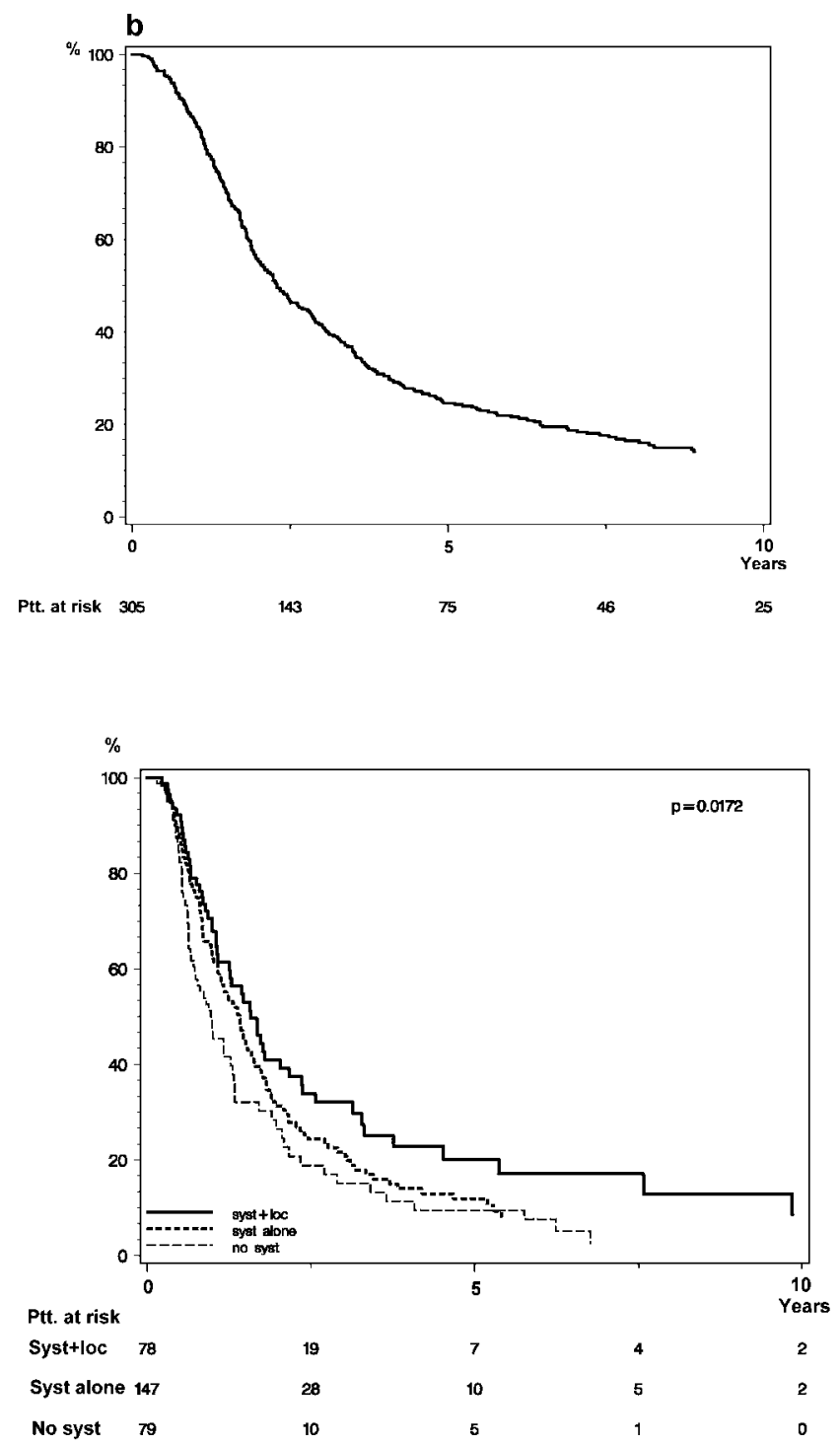